\title{
Nanoparticle Production by UV Irradiation of Combustion Generated Soot Particles
}

\author{
Christopher B. Stipe ${ }^{1}$, Jong Hyun Choi ${ }^{1}$, Donald Lucas ${ }^{2 *}$, Catherine P. Koshland ${ }^{3}$, and Robert F. \\ Sawyer ${ }^{1}$ \\ ${ }^{1}$ Mechanical Engineering Department \\ University of California at Berkeley \\ ${ }^{2}$ Environmental Energy Technologies Division \\ Lawrence Berkeley National Laboratory \\ ${ }^{3}$ School of Public Health \\ University of California at Berkeley
}

Keywords: Soot, Particles, Nanoparticles, Photofragmentation, Ablation

* Corresponding Author:

Donald Lucas

70-108B

LBNL

Berkeley, CA 94720

D_lucas@lbl.gov

510-486-7002

510-486-7303 (FAX) 


\begin{abstract}
Laser ablation of surfaces normally produce high temperature plasmas that are difficult to control. By irradiating small particles in the gas phase, we can better control the size and concentration of the resulting particles when different materials are photofragmented. Here, we irradiate soot with $193 \mathrm{~nm}$ light from an ArF excimer laser. Irradiating the original agglomerated particles at fluences ranging from 0.07 to $0.26 \mathrm{~J} / \mathrm{cm}^{2}$ with repetition rates of 20 and $100 \mathrm{~Hz}$ produces a large number of small, unagglomerated particles, and a smaller number of spherical agglomerated particles. Mean particle diameters from 20 to $50 \mathrm{~nm}$ are produced from soot originally having a mean electric mobility diameter of $265 \mathrm{~nm}$. We use a non-dimensional parameter, called the photon/atom ratio (PAR), to aid in understanding the photofragmentation process. This parameter is the ratio of the number of photons striking the soot particles to the number of the carbon atoms contained in the soot particles, and is a better metric than the laser fluence for analyzing laser-particle interactions. These results suggest that UV photofragmentation can be effective in controlling particle size and morphology, and can be a useful diagnostic for studying elements of the laser ablation process.
\end{abstract}




\section{Introduction}

Laser ablation of surfaces is a complex system involving heating, photochemistry, and photophysics that has been studied by numerous research groups since the invention of high power lasers. The species produced during laser irradiation of a particle or bulk surface depend on the fluence, duration, and wavelength of the laser pulse, in addition to the original chemical composition of the particle or surface (Miller and Haglund 1998).

Understanding the laser-material interactions at a fundamental level can improve particle production techniques as well as laser diagnostics based on photofragmentation or disintegration. Nanoparticles produced by laser ablation of surfaces are often fractal agglomerates, an undesirable trait for some nanoparticle applications, and the process is difficult to scale. Numerous researchers have investigated the production of nanoparticles by ablating bulk surfaces (e.g., Geohegan, Puretzky et al. 1999; Jeong, Borisov et al. 1999; Ullmann, Friedlander et al. 2002); the references cited focus on methods developed to control the resulting nanoparticle size and extent of agglomeration. Unlike simple carbon black nanoparticles, produced by low-technology methods such as low-temperature combustion, more advanced nanoparticle applications, such as those used in the electronics and biomedical fields, often require unagglomerated particles of a controlled size.

Laser fragmentation of small particles in the gas phase has received much less attention. When micrometer sized particles deposited on a plate or in an aerosol stream are irradiated by a pulsed beam at 248 nm (Becker, Brock et al. 1998; Nichols, Keto et al. 2001), smaller unagglomerated spherical particles form during and after laser irradiation, with the small particle formation attributed to homogeneous nucleation of the gas phase species ejected from the large irradiated particle. 
We found that there are significant differences in the laser-material interactions when the particles are in the submicron range - the particles do not exhibit incandescence, and the broadband background emission is virtually absent (Stipe, Lucas et al. 2003; Stipe, Higgins et al. 2002). Here, we modify soot particles from a laboratory diffusion flame using UV photons. The initial soot agglomerates irradiated by $193 \mathrm{~nm}$ laser pulses produce spherical, unagglomerated nanoparticles of a controlled diameter. This technique shows promise for producing a high concentration of nanoparticles for use in advanced engineering applications and for better understanding laser-particle interactions.

\section{Experimental Apparatus}

The experimental apparatus is shown in Figure 1. Combustion generated particles are created by an inverted, co-flow, non-premixed burner described previously (Stipe, Higgins et al. 2002). Methane flows at 1.27 liters per minute (lpm) through a $2 \mathrm{~cm}$ diameter center jet surrounded by a shroud of $19 \mathrm{lpm}$ of co-flow air enclosed in a $5 \mathrm{~cm}$ diameter quartz tube. The methane and air were at atmospheric pressure and $293 \mathrm{~K}$. The flame is roughly $30 \mathrm{~cm}$ in length. Air injected $4 \mathrm{~cm}$ downstream of the flame tip at $25 \mathrm{lpm}$ dilutes and cools the soot and exhaust gases exiting the quartz tube. The soot and exhaust gases then flow through a $140 \mathrm{~cm}$ stainless steel tube to enhance mixing. An ejector pump further upstream extracts $2 \mathrm{lpm}$ of flow from the stainless steel tube and further dilutes the flow by a factor of 11 . The flow passes through a diffusion dryer to remove water and through a diffusion denuder to remove unburned gas phase hydrocarbons.

Of the $22 \mathrm{lpm}$ exiting the ejector pump, half flows to a mixing chamber, where the number particles drops and the mean particle diameter increases through collisions. The flow then travels through a BGI cyclone (model KTL GK2.05), which removes particles larger than 
$900 \mathrm{~nm}$ in diameter. These large particles are removed to ensure the particles flowing to the laser interrogation region are measured by a scanning mobility particle sizer (SMPS), which at its inlet has an $800 \mathrm{~nm}$ particle impactor. After exiting the cyclone, a valve controls the flow such that $0.3 \mathrm{lpm}$ flows to a $1 \mathrm{~cm}^{2}$ quartz cuvette in the laser interrogation region, and the remaining gas flows to an exhaust hood. The quartz cuvette is a standard $1 \mathrm{~cm}$ path length absorption cell used for UV/visible spectroscopy, with the closed end removed to allow flow-through operation.

A Lambda Physik LPX210i ArF excimer laser photofragments the particles in the quartz cell. The $20 \mathrm{~ns}$ beam of $193 \mathrm{~nm}$ photons is slightly focused by a $38.1 \mathrm{~mm}$ diameter, $128 \mathrm{~mm}$ focal length plano-convex lens. The spot size at the surface of the cuvette is $12 \mathrm{~mm}$ in width by 4 $\mathrm{mm}$ in height. The cuvette is $12 \mathrm{~mm}$ by $12 \mathrm{~mm}$ by $40 \mathrm{~mm}$ in height with a wall thickness of 1 $\mathrm{mm}$, so the light intercepts $\sim 90 \%$ of the particles in the beam footprint. Laser power is measured with a Gentec joulemeter. The front surface of the cuvette absorbs or scatters approximately $20 \%$ of the incident $193 \mathrm{~nm}$ photons; the fluences presented are corrected for this loss.

Particles exiting the cuvette travel to the SMPS for size distribution measurement. The SMPS consists of a Differential Mobility Analyzer (DMA) (TSI model 3071A), which separates a fraction of the total polydisperse particles based on the electric mobility diameter of the particles and a Condensation Particle Counter (CPC) (TSI model 3025A) that quantifies the number concentration of the size selected particles.

A $47 \mathrm{~mm}$ diameter Whatman anodisc membrane filter between the quartz cell and the SMPS inlet captures the particles for analysis by SEM. A layer of an Au/Pd alloy coats the particles and filter for improved imaging by enhancing the conductivity. Soot particles are collected on $37 \mathrm{~mm}$ diameter, $2 \mu \mathrm{m}$ pore size, SKC Inc. Teflon membrane filters for mass analysis. A Millipore Corp. filter cartridge holds the filter for particle collection. Loading the 
filters for one hour deposits adequate mass ( $\sim 30 \mu \mathrm{g})$ for measurement with a Cahn Model 21 Automatic Electrobalance. The electrobalance is accurate to within $\pm 2 \mu \mathrm{g}$ for the $20 \mathrm{mg}$ range setting.

\section{Results and Discussion}

\section{Single Shot Irradiation}

Figure 2 shows the soot particle size distribution as a function of laser fluence irradiated at a laser repetition rate of $20 \mathrm{~Hz}$. The non-fragmented particles, shown as open circles, have a mean electric mobility diameter of $270 \mathrm{~nm}$, a number concentration of $4.5 \cdot 10^{5} \mathrm{~cm}^{-3}$, a surface area concentration of $3.3 \cdot 10^{10} \mathrm{~nm}^{2} / \mathrm{cm}^{3}$, and a volume concentration of $9.5 \cdot 10^{11} \mathrm{~nm}^{3} / \mathrm{cm}^{3}(\sim 1$ $\mathrm{ppb}$ ), as measured by the SMPS system (the SMPS determines only mobility diameter and several assumptions are inherent in determining volumes and surface areas; we use these values to readily compare changes in these parameters). The concentration, assuming all carbon atoms in the particles are converted to gas phase atoms, is $4 \cdot 10^{-6} \mathrm{C}$ atoms/air molecule (4 ppm). The velocity of the particle-laden flow through the quartz tube is $5 \mathrm{~cm} / \mathrm{s}$, so at a laser repetition rate of $20 \mathrm{~Hz}$ and a vertical beam height of $0.4 \mathrm{~cm}$, on average 1.6 laser pulses hit each particle. Table 1 presents pertinent data for the single shot $(20 \mathrm{~Hz})$ and multiple shot $(100 \mathrm{~Hz})$ experiments.

We developed a parameter called the photon-atom ratio (PAR) to aid in understanding the laser-particle interaction. PAR is the ratio of the number of photons striking the particle to the number of atoms in the particle. When the photons have the same energy as the average bond energy in a particle, the PAR number must be equal or greater than unity for complete disintegration. The more commonly used laser fluence is not as useful for describing the energy 
balance of systems composed of differing particle sizes, since the ratio of the energy incident on the particle to the volume of the particles is not constant over all particle sizes.

The PAR number is corrected for various factors, including the absorption coefficient of the particle material, the enthalpy of atomization (the energy required to convert solid graphite to carbon atoms is $716 \mathrm{~kJ} / \mathrm{mole}$ (Pierson 1993)), and the energy of the photons. For soot irradiated by $193 \mathrm{~nm}$ photons, the energy in a single photon is larger than the energy of a $\sigma$ bond between carbon atoms (620 vs. $524 \mathrm{~kJ} / \mathrm{mole}$ ) (Pierson 1993), so each photon can break at least one bond. In comparison, the $\pi$ bonds are much weaker ( $7 \mathrm{~kJ} / \mathrm{mole}$ ) (Pierson 1993).

Accurately determining the fraction of incident energy absorbed by an agglomerate particle is difficult, as it depends on the shape of the particle, the optical properties of the particle, and the wavelength of the incident electromagnetic wave. Large variations in the measured optical properties of soot exist in the literature (Michelsen 2003). We use 0.815-0.950i for the refractive index of graphite at $193 \mathrm{~nm}$ (Edoh 1983). Since the laser wavelength is similar to the diameter of the particles, Mie scattering theory is employed to determine the absorption and scattering coefficients (Modest 1993). Another difficulty arises due to the agglomerated shape of the particles. In most laser based measurements of particles, the primary particles are assumed to act as independent absorbing or scattering media, even when combined in an agglomerate particle (Smallwood, Snelling et al. 2001; Smallwood, Clavel et al. 2002; Michelsen 2003). However, other studies have shown that this assumption is not valid, as even agglomerate particles composed of two primary particles scatter differently than the two individual particles (Xu and Wang 1998). The extent of the difference depends on the relative size of the primary particle to the agglomerated particle and the distance between the centers of the primary particles. 
The spherical primary soot particles are roughly $40 \mathrm{~nm}$ in diameter and absorb approximately 90\% of the incident $193 \mathrm{~nm}$ light (Edoh 1983), using the complex index of refraction at $193 \mathrm{~nm}$ of graphite perpendicular to the graphitic layers (0.815-0.950i). The mean diameter of the agglomerate is $270 \mathrm{~nm}$, which scatters approximately 65\% of $193 \mathrm{~nm}$ light assuming it acts as an independent sphere. It is likely that a fraction of the primary particles act as independent spheres, while others have coalesced and act in a combined fashion. We assume that the soot particles absorb approximately $80 \%$ of incident light at $193 \mathrm{~nm}$, which is roughly the average of the independent and dependent values. It should be noted that none of our results and conclusions depend on the exact values of these parameters.

Irradiation of the particles, as seen in Fig. 2, generates a new mode of particles significantly smaller in diameter than the original particles with a number concentration almost two orders of magnitude higher. For irradiation at PARs from 0.06 to 0.13 (fluences from 0.07 to $0.14 \mathrm{~J} / \mathrm{cm}^{2}$ ), the number concentration and mean diameter of the small mode of particles increases. The total volume concentration remains constant because most of the particles are not disintegrated. The PAR numbers indicate that the particles only absorb a small fraction (6 to $13 \%$ ) of the total energy necessary to completely convert the original particles to atomic species. At a PAR of 0.06, most of the original large and more massive particles remain after irradiation, as shown in Fig. 3.

The trend of increasing mean diameter and number concentration for the small mode of particles does not continue for PAR numbers above 0.13. For PAR numbers from 0.17 to 0.25 (fluences from 0.18 to $0.26 \mathrm{~J} / \mathrm{cm}^{2}$ ) at $20 \mathrm{~Hz}$, the mean diameter of the new peak remains relatively unchanged, while the total volume concentration of the particles decreases by 
approximately $30 \%$, as measured by the SMPS. Few of the original particles larger than the mean remain, as seen in Fig. 4.

\section{Multiple Shot Irradiation}

To study higher PAR values, the laser repetition rate is increased from 20 to $100 \mathrm{~Hz}$ because increasing the fluence of a single pulse damages the quartz cell. The same original size distribution of soot particles are irradiated over the same range of fluences $\left(0.07\right.$ to $\left.0.26 \mathrm{~J} / \mathrm{cm}^{2}\right)$ as in the $20 \mathrm{~Hz}$ experiment, and the results are shown in Fig. 5. At the higher repetition rate, each particle is irradiated by approximately 8 laser shots. Adding energy to a particle in multiple pulses likely causes a different extent of disintegration than adding the same amount of energy in one pulse since the time between pulses allows for physical processes and chemical reactions to occur for both the particle and its fragmentation products. Previous results using a two laser experiment revealed that the laser induced modifications occur within 500 ns after the laser pulse, which is a short time compared to the $1 \mathrm{~ms}$ between laser pulses for this experiment (Stipe, Lucas et al. 2003). The state of the particles after each laser pulse is unknown; therefore, the overall PAR is determined by simply multiplying the PAR for a single shot by the number of shots.

The trend of decreasing volume concentration with increasing PAR observed for the highest PARs at $20 \mathrm{~Hz}$ continues at $100 \mathrm{~Hz}$. In this case, both the number and mean diameter of the small particles decrease with increasing PAR. For PARs from 0.32 to 1.23 , the photons increasingly obliterate the original particle size distribution. The mean diameter of the newly created peak decreases from 50 to $18 \mathrm{~nm}$ over this range for PARs, with 95\% of the original volume lost at the highest PAR. 
Filter mass measurements of the non-irradiated particles and particles irradiated at 100 $\mathrm{Hz}$ and a PAR of $1.23\left(0.26 \mathrm{~J} / \mathrm{cm}^{2}\right)$ confirms the loss of volume; $77 \pm 7 \%$ of the original mass is lost when irradiated. These measurements are in reasonable agreement with the SMPS values, especially since the two techniques detect different properties, and the SMPS volume calculations involve several assumptions about particle size and electrical mobility.

\section{SEM Images}

The soot particles are examined visually using SEM; Fig. 6 shows two images. Nonirradiated soot particles, shown in 6(a), confirm the agglomerate shape of the soot composed of roughly $40 \mathrm{~nm}$ diameter primary particles with a fractal dimension near the accepted value of 1.8 (Wagner 1979).

The effect of laser irradiation is shown in 6(b). The particles irradiated with a PAR of $0.85\left(0.18 \mathrm{~J} / \mathrm{cm}^{2}, 100 \mathrm{~Hz}\right)$ have changed in both size distribution and fractal dimension. There are two distinct types of particles: small spherical particles with a diameter near $40 \mathrm{~nm}$ and clusters larger than $1 \mu \mathrm{m}$. The large clusters are nearly spherical and appear to be composed of agglomerated primary particles.

\section{Oxidation}

The volume of the soot particles remaining after laser irradiation decreases with increasing PAR. Two oxidation pathways could reduce the soot volume concentration: the direct oxidation of species from the surface of the soot particles and the oxidation of carbon atoms and other reactive gas phase species liberated from the particles by laser irradiation. Measuring the increase in the $\mathrm{CO}_{2}$ concentrations could confirm the mass loss due to oxidation, but the 
background concentration of $\mathrm{CO}_{2}$ in the diluted burner exhaust is too large $(\sim 1 \%)$ compared to $\mathrm{CO}_{2}$ produced by soot or carbon atom oxidation $(\sim 4 \mathrm{ppm})$. Soot begins to oxidize at roughly $600^{\circ} \mathrm{C}$ at atmospheric pressure (Stanmore, Brilhac et al. 1999; Stanmore, Brilhac et al. 2001). Direct oxidation of the solid soot by $\mathrm{O}_{2}$ is thus not likely as the temperature of the surrounding bath gases or the particles themselves is not high enough, since the particles do not exhibit incandescence. However, surface oxidation by oxygen atoms and ozone is possible.

Photolysis of oxygen molecules by $193 \mathrm{~nm}$ photons produces oxygen atoms (Lee and Hanson 1986) and ozone from the reaction of atomic oxygen with $\mathrm{O}_{2}$. The concentration of oxygen atoms, produced by photolysis of $\mathrm{O}_{2}$ in air, is on the same order of magnitude as the concentration of carbon atoms produced by irradiating the soot particles $(\sim 4 \mathrm{ppm})$, while the concentration of molecular oxygen at $20.9 \%$ is over four orders of magnitude larger than the carbon atom concentration. Molecular oxygen likely does not significantly oxidize the particles, but it will oxidize reactive carbon species ejected by laser irradiation. In addition, the atomic oxygen and ozone may oxidize either the particle directly or ejected species, both leading to a reduction in volume.

We lowered the oxygen concentration to $1.8 \%$ by diluting the flame exhaust products with nitrogen instead of air in the ejector pump; an oxygen free environment was not possible since the burner operated with excess oxygen. A comparison of original soot particles, soot particle irradiated in $20.9 \% \mathrm{O}_{2}$, and soot particles irradiated in $1.8 \% \mathrm{O}_{2}$ is shown in Fig. 7. The non-irradiated soot size distribution is the same for both diluents $\left(265 \mathrm{~nm}, 4.5 \cdot 10^{5} \mathrm{~cm}^{-3}\right.$, and $\left.9.5 \cdot 10^{11} \mathrm{~nm}^{3} / \mathrm{cm}^{3}\right)$. The particles fragmented in $20.9 \% \mathrm{O}_{2}$ at a PAR of $0.95\left(0.2 \mathrm{~J} / \mathrm{cm}^{2}, 100 \mathrm{~Hz}\right)$ have a number concentration of $1.9 \cdot 10^{6} \mathrm{~cm}^{-3}$ and a volume of $6.8 \cdot 10^{10} \mathrm{~nm}^{3} / \mathrm{cm}^{3}$. Particles fragmented in $1.8 \% \mathrm{O}_{2}$ at the same PAR have 3 times as many particles as the air case, and a 
volume of $1.3 \cdot 10^{11} \mathrm{~nm}^{3} / \mathrm{cm}^{3}$. More volume is lost when photofragmenting in air (93\%) compared to the reduced oxygen case (85\%), but it is not clear that this difference is significant.

\section{Particle Disintegration}

Particle disintegration depends on the wavelength, fluence, time scale of the pulse, and chemical composition of the irradiated material. Photochemical bond breaking likely dominates the disintegration under the conditions studied here as the $193 \mathrm{~nm}$ photons are energetic enough to directly break bonds and liberate atomic and molecular species from the original soot particles. Even photons absorbed within the particle lead to internal bond breaking.

Thermal ablation is not an important disintegration mechanism for these experiments. The fluences used here $\left(\leq 0.26 \mathrm{~J} / \mathrm{cm}^{2}\right.$ or $\left.13 \mathrm{MW} / \mathrm{cm}^{2}\right)$ are below those generally associated with thermal ablation and plasma formation. A laser ablation study at $193 \mathrm{~nm}$ of graphite by Mechler et al. (Mechler, Heszler et al. 2000) found a threshold of $1 \mathrm{~J} / \mathrm{cm}^{2}\left(66 \mathrm{MW} / \mathrm{cm}^{2}\right.$ ) for removal of a detectable amount of material from a surface. However, the study was conducted on bulk graphite, and material properties and photon absorption characteristics at the nanometer scale differ from those of bulk materials (Sonwane, Mintmire et al. 2003). Laser ablation studies of particles at $193 \mathrm{~nm}$ by Thomson and Murphy (Thomson and Murphy 1993) provides additional evidence that thermal ablation is not important here. They determined the threshold for producing a detectable level of ionized atomic and molecular species at $0.25 \mathrm{~J} / \mathrm{cm}^{2}\left(25 \mathrm{MW} / \mathrm{cm}^{2}\right)$ for micrometer-sized particles. They suggested the threshold for plasma formation in particle laden air is approximately $5 \mathrm{~J} / \mathrm{cm}^{2}\left(500 \mathrm{MW} / \mathrm{cm}^{2}\right)$. As mentioned previously, an insignificant amount of photon energy is converted to heat in the particles as no blackbody radiation (incandescence) is observed. 


\section{Small Particle Generation}

For the lowest three PARs $(0.06,0.10$, and 0.13$)$ at $20 \mathrm{~Hz}$, the number concentration of small particles increases with increasing PAR, while the total volume concentration remains roughly constant. The energy absorbed by the particles is only 6 to $13 \%$ of the energy necessary for full atomization. Therefore, some particle fragments, clusters, or large molecules must remain after irradiation. The number concentration of new particles increases by almost 25 times over this range of PARs, without losing a significant amount of volume by oxidation.

For PARs equal to and above 0.17 at $20 \mathrm{~Hz}$, the volume concentration of particles decreases with increasing PAR, while the number concentration of particles remains relatively unchanged. The increased number of photons incident on the particles liberates more material from the surface of the particles that are subsequently oxidized, leading to the reduction in overall volume. The trend of decreasing volume is also observed for all PARs generated by irradiating the particles with 8 shots $(100 \mathrm{~Hz})$. Here, the mean diameter of the particle size distribution also decreases as even more material is photolyzed. The volume reduction of soot at high PARs measured by the SMPS system is confirmed by filter mass measurements. In other experiments we irradiated $\mathrm{NaCl}$, a material not expected to significantly oxidize. The initial volume was conserved over all photolysis conditions, giving confidence that the loss of volume for soot is attributable to oxidation. In the results here, most of the particle mass is lost when irradiated at a high PAR, suggesting that species liberated at lower PARs will also oxidize.

The highest two PARs at $100 \mathrm{~Hz}$ are larger than unity, where the original particles absorb more energy than necessary for complete atomization. Thus, the disintegration is not perfectly efficient. The energy is delivered in 8 laser pulses, and each laser pulse likely alters the particle surface area to volume ratio and the parameters used to calculate the PAR. 
The liberated species may form new particles through homogeneous nucleation or by heterogeneously nucleating with remaining particle fragments. Nichols et al. (Nichols, Keto et al. 2001) and Becker et al. (Becker, Brock et al. 1998) produced nanoparticles by irradiating micron-sized metal and glass particles. They suggest the gas phase species condense to form the small particles as the ablation plume cools. Their initial particles are 1 to $10 \mu \mathrm{m}$ in diameter and are irradiated at fluences up to $15 \mathrm{~J} / \mathrm{cm}^{2}\left(1.5 \cdot 10^{9} \mathrm{~W} / \mathrm{cm}^{2}\right)$ at $248 \mathrm{~nm}$. The fluence is sufficient to produce a plasma, and the particle diameters are larger than the absorption depth of the laser pulse. Thus, they attribute the particle disintegration to shockwave formation within the particle.

At lower PARs in our experiment, increasing the fluence liberates more gas phase species, making more material available for nucleation. The increasing number of particles formed as the PAR increases implies the original particles are not fully disintegrated by one laser pulse and the rate of particle production exceeds the rate of oxidation. At PARs produced by multiple laser shots, $10 \mathrm{~ms}$ separates each shot. Between pulses, some of the gas phase species nucleate to form new particles, while those at the boundary with the surrounding air oxidize. Subsequent laser pulses further disintegrate the original particles, photolyze the newly nucleated particles, and create more atomic oxygen and ozone. Under the multiple shot condition, the species spend more time in the gas phase, leading to increased loss of material by oxidation.

To assess the likelihood of the nucleation formation pathway, the timescales for particle formation and oxidation of the gas phase species are estimated. A simplistic model based on collisional theory is used to estimate the time necessary to form $40 \mathrm{~nm}$ particles. The number concentration of the original non-irradiated particles is approximately $4.5 \cdot 10^{5} \mathrm{~cm}^{-3}$. In the limit of complete fragmentation of the particle and a homogeneous distribution of products, the time needed exceeds $0.1 \mathrm{sec}$. Assuming a $265 \mathrm{~nm}$ particle is fully disintegrated and the resulting 
carbon atoms expand to fill a volume of 100 particle diameters, the nucleation time for a $40 \mathrm{~nm}$ particle is roughly $110 \mu$ s, still much longer than the 500 ns time frame observed for particle modification in our previous experiments. Thus, it is likely that the plume of products is confined to a smaller volume.

The oxidation rate of ejected carbon species depends on the mixing time between the carbon atoms and the surrounding oxygen molecules. The complexities of the particle disintegration and motion of the ejected species make estimating the mixing time difficult. As an approximation, the time for an $\mathrm{O}_{2}$ molecule to diffuse the 100 particle diameters, used in the previous example, is $20 \mu$ s. Therefore, if the concentration of carbon atoms is uniformly mixed with air after disintegration, it is unlikely that a $40 \mathrm{~nm}$ particle will nucleate before the carbon atoms oxidize. However, if the ejected species expand 100 times the original particle diameter, the diffusion timescale and nucleation timescale are roughly equivalent.

\section{Large Particle Generation}

Modeling results of particle agglomeration by Schaefer (Schaefer 1988; Schaefer and Hurd 1990) are used to predict the collisional environment producing particles of specific fractal dimensions. For example, soot particles generated in a flame, with a fractal dimension of 1.8, are produced by cluster-cluster agglomeration in a diffusion limited regime. The larger clusters observed in the SEM images of the irradiated particles, with a fractal dimension near 3, form under particle-cluster agglomeration in a reaction limited regime.

Ivanenko (Ivanenko, Lebovka et al. 1999) found that reaction limited agglomeration occurs between particles and clusters of the same charge. Since charged particles repel each other, only particles colliding with adequate energy to overcome the electrostatic barrier agglomerate. Particles colliding with a preexisting cluster of particles of the same charge imbed 
more deeply into cluster, forming the spherical shape of the cluster. This agglomeration mechanism, also called "Eden" growth, can produce the large clusters in Fig. 6(b). While a plasma is not observed in this system, some ionization of gas phase atoms, molecules, or particle fragments is likely occurring.

It is improbable that the large particle clusters form by nucleation or through rearrangement of the primary particles. The primary particles are distinctively seen in the larger particle clusters. If the large clusters were formed by nucleation, a smoother outer boundary would be observed. Rearrangement of primary particles occurs when weakly attached primary particles restructure to reduce the Gibbs free energy of the cluster (Friedlander 2000). This is unlikely as the number of primary particles in a spherical cluster $1 \mu \mathrm{m}$ in diameter composed of $40 \mathrm{~nm}$ primary particles is approximately 15,000, while the number of primary particles in the original fractal particles is only around 300 .

\section{Conclusions}

$193 \mathrm{~nm}$ laser irradiation of combustion generated soot produces unagglomerated nanoparticles and spherical agglomerated particles whose size and number are controlled by varying the laser fluence and repetition rate. The smaller particles vary continuously in size from 20 to $50 \mathrm{~nm}$, indicating that the process is not simply a fragmentation of the aggolomerated soot into primary particles. The smaller particles are observed even at low laser fluences, where there is insufficient energy to completely vaporize the soot. We use a non-dimensional parameter, called the photon/atom ratio (PAR), to aid in understanding the photofragmentation process. This parameter is the ratio of the number of photons striking the soot particles to the number of the carbon atoms contained in the soot particles, and is a better metric than the laser fluence for 
analyzing laser-particle interactions. As the PAR values increase more of the original volume of particles is lost by gas phase oxidation. SEM images also show drastic changes in the fractal dimension of the soot agglomerates when irradiated. At high PAR values, large spherical clusters form by the agglomeration of charged primary particles. These results suggest that UV photofragmentation can be effective in controlling particle size and morphology.

\section{Acknowledgements}

This work was supported by the Environmental Health Sciences Superfund Basic Research Program (Grant Number P42ESO47050-01) from the National Institute of Environmental Health Sciences, NIH, with funding provided by the EPA and the Toxic Substances Research and Teaching Program. Its contents are solely the responsibility of the authors and do not necessarily represent the official views of NIEHS, NIH, or EPA.

\section{References}

Becker, M. F., J. R. Brock, H. Cai, D. E. Henneke, J. W. Keto, J. Lee, W. T. Nichols and H. D. Glicksman, 1998. Metal Nanoparticles Generated by Laser Ablation. Nanostructured Mat. 10(5): 853-863.

Edoh, O., 1983. Optical Properties of Carbon from the Far Infrared to the Far Ultraviolet. Physics:University of Arizona 99.

Friedlander, S. K., 2000. Smoke, Dust, and Haze: Fundamentals of Aerosol Dynamics. New York, NY, Oxford University Press, 407. 
Geohegan, D. B., A. A. Puretzky and D. J. Rader, 1999. Gas-phase Nanoparticle Formation and Transport During Laser Deposition of Y1Ba2Cu3O7. Appl. Phys Lett. 74(25): 3788-3790.

Ivanenko, Y. V., N. I. Lebovka and N. V. Vygornitskii, 1999. Eden Growth Model for Aggregation of Charged Particles. Eur. Phys. J. B 11: 469-480.

Jeong, S. H., O. V. Borisov, J. H. Yoo, X. L. Mao and R. E. Russo, 1999. Effects of Particle Size Distribution on Inductively Coupled Plasma Mass Spectrometry Signal Intensity During Laser Ablation of Glass Samples. Anal. Chem. 71(22): 5123-5130.

Lee, J., M. F. Becker and J. W. Keto, 2001. Dynamics of Laser Ablation of Microspheres Prior to Nanoparticle Generation. J. Appl. Phys. 89(12): 8146-8152.

Lee, M. P. and R. K. Hanson, 1986. Calculations of O2 Absorption and Fluorescence at Elevated Temperatures for a Broadband Argon-Fluoride Laser Source at 193nm. J. Quant. Spectrosc. Radiat. Transfer 36(5): 425-440.

Mechler, A., P. Heszler, Z. Marton, M. Kovacs, T. Szorenyi and Z. Bor, 2000. Raman Spectroscopic and Atomic Force Microscopic Study of Graphite Ablation at 193 and 248 nm. Appl. Surf. Sci. 154-155: 22-28.

Michelsen, H. A., 2003. Understanding and Predicting the Temporal Response of Laser-Induced Incandescence from Carbonaceous Particles. J. of Chem. Phys. 118(15).

Miller, J. C. and R. F. Haglund, 1998. Laser Ablation and Desorption In Experimental Methods in the Physical Sciences. New York, Academic Press, 30.

Modest, M. F., 1993. Radiative Heat Transfer. New York, McGraw-Hill, 860.

Nichols, W. T., J. W. Keto, D. E. Henneke, J. R. Brock, G. Malyavanatham, M. F. Becker and H. D. Glicksman, 2001. Large-scale Production of Nanocrystals by Laser Ablation of Microparticles in a Flowing Aerosol. Appl. Phys. Lett. 78(8): 1128-1130. 
Pierson, H. O., 1993. Handbook of Carbon, Graphite, Diamond and Fullerenes - Properties, Processing and Applications. Park Ridge, New Jersey, William Andrew Publishing/Noyes, 399.

Schaefer, D. W., 1988. Fractal Models and the Structure of Materials. MRS Bulletin 13: 22-27. Schaefer, D. W. and A. J. Hurd, 1990. Growth and Structure of Combustion Aerosols. Aerosol Sci. and Technol. 12: 876-890.

Smallwood, G. J., D. Clavel, D. Gareau, R. A. Sawchuk, D. R. Snelling, P. O. Witze, B. Axelsson, W. D. Bachalo and O. L. Gulder, 2002. Concurrent Quantitative Laser-Induced Incandescence and SMPS Measurements of EGR Effects on Particulate Emissions from a TDI Diesel Engine. SAE Technical Paper 2002-01-2715: 1-16.

Smallwood, G. J., D. R. Snelling, F. Liu and O. L. Gulder, 2001. Clouds Over Soot Evaporation: Errors in Modeling Laser-Induced Incandescence of Soot. Transactions of the ASME 123: 814-818.

Sonwane, C. G., J. Mintmire and M. R. Zachariah, 2003. Molecular Dynamics Simulations of the Interfacial Properties and Reactivity of Bare and Oxide Coated Aluminum Nanoclusters. Proceedings of the 3rd Joint Meeting of the U.S. Sections of The Combustion Institute. Stanmore, B. R., J. F. Brilhac and P. Gilot, 1999. The Ignition and Combustion of Cerium Doped Diesel Soot. SAE Paper 1999-01-0115: 1-7.

Stanmore, B. R., J. F. Brilhac and P. Gilot, 2001. The oxidation of soot: a review of experiments, mechanisms, and models. Carbon 39: 2247-2268.

Stipe, C. B., B. S. Higgins, D. Lucas, C. P. Koshland and R. F. Sawyer, 2002. Soot Detection Using Excimer Laser Fragmentation Fluorescence Spectroscopy. 29th Symposium (Int.) on Combustion, Sapporo, Japan. 
Stipe, C. B., D. Lucas, R. F. Sawyer and C. P. Koshland, 2003. Two Laser UV Photofragmentation of Soot Particles. 3rd Joint Meeting of the U.S. Sections of The Combustion Institute, Chicago, Illinois, USA.

Thomson, D. S. and D. M. Murphy, 1993. Laser-Induced Ion Formation Thresholds of Aerosol Particles in a Vacuum. Appl. Opt. 32(33): 6818-6826.

Ullmann, M., S. K. Friedlander and A. Schmidt-Ott, 2002. Nanoparticle Formation by Laser Ablation. J. Nanoparticle Res. 4: 499-509.

Wagner, H. G., 1979. Soot Formation in Combustion. Seventeenth Symposium (Int.) on Combustion, The University of Leed, U.K..

Xu, Y. and R. T. Wang, 1998. Electromagnetic Scattering by an Aggregate of Spheres: Theoretical and Experimental Study of the Amplitude Scattering Matrix. Phys. Rev. E 58(3): 3931-3948. 


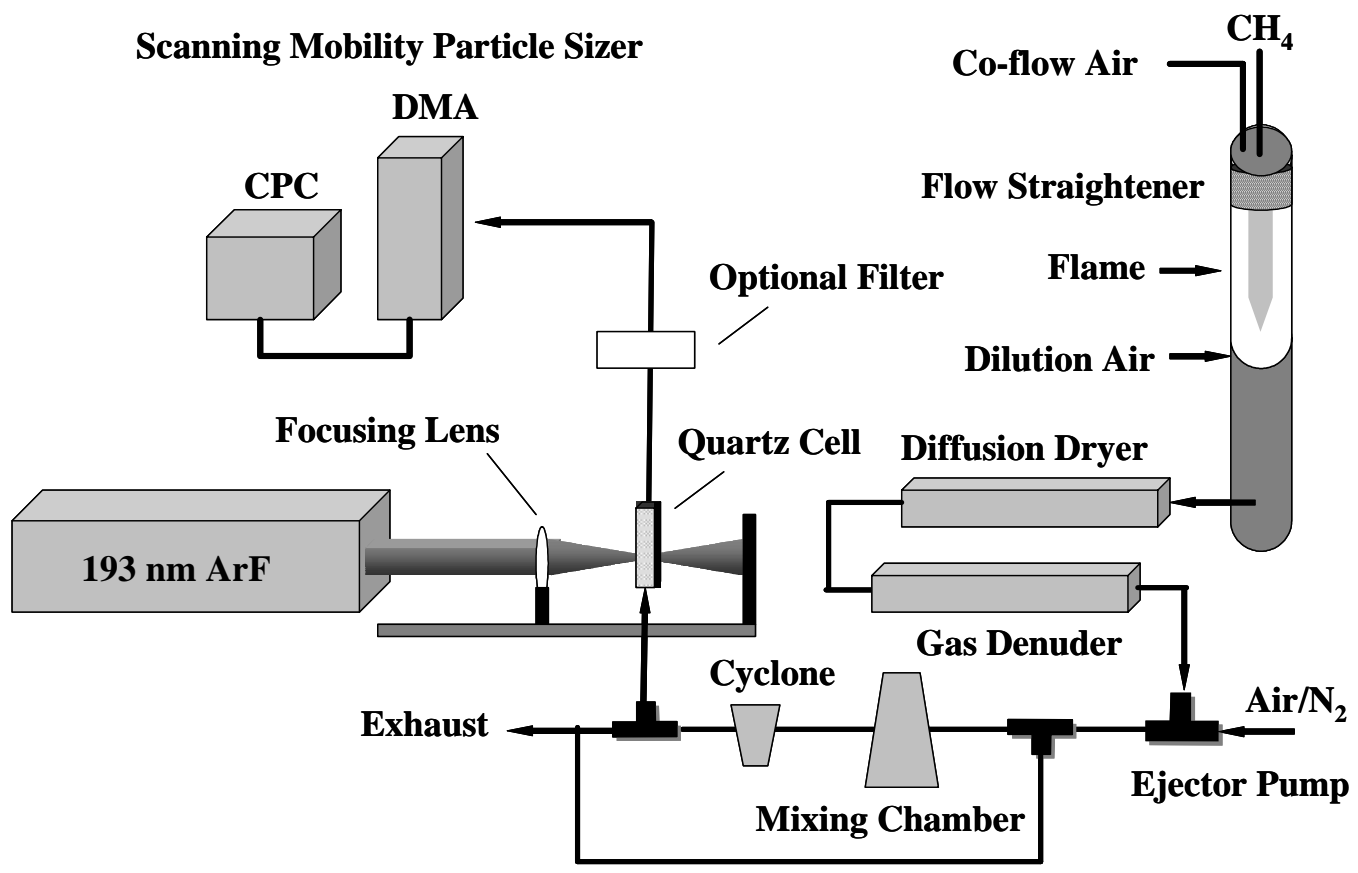

Figure 1: Experimental apparatus. 


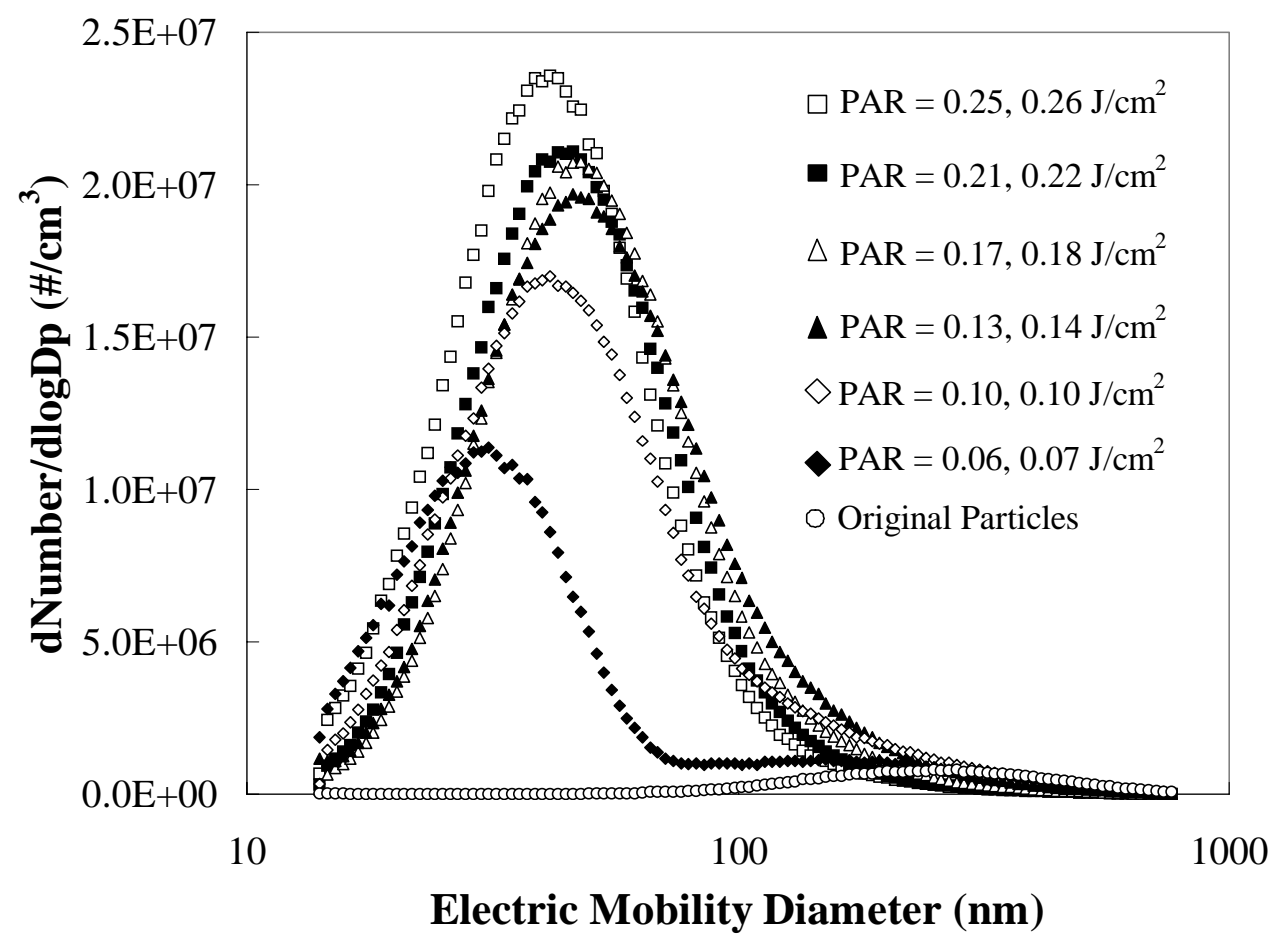

Figure 2: Size distributions of soot particles fragmented with PAR values from 0.06 to 0.25 (fluences of 0.07 to $0.26 \mathrm{~J} / \mathrm{cm}^{2}$ ) by $193 \mathrm{~nm}$ photons at $20 \mathrm{~Hz}$ (1.6 shots/particle). The original particle size distribution is shown in open circles. 


\begin{tabular}{|c|c|c|c|c|c|c|c|}
\hline $\begin{array}{l}\text { Fluence } \\
\left(\mathrm{J} / \mathrm{cm}^{2}\right) \\
\end{array}$ & $\begin{array}{c}\text { Repetition } \\
\text { Rate } \\
(\mathrm{Hz}) \\
\end{array}$ & $\begin{array}{c}\text { Total } \\
\text { Diameter } \\
(\mathbf{n m}) \\
\end{array}$ & $\begin{array}{c}\text { Small } \\
\text { Diameter } \\
(\mathbf{n m}) \\
\end{array}$ & $\begin{array}{c}\text { Number } \\
\text { Concentration } \\
\left(\# / \mathrm{cm}^{3}\right) \\
\end{array}$ & $\begin{array}{c}\text { Surface Area } \\
\text { Concentration } \\
\left(\mathrm{nm}^{2} / \mathrm{cm}^{3}\right)\end{array}$ & $\begin{array}{c}\text { Volume } \\
\text { Concentration } \\
\left(\mathrm{nm}^{3} / \mathrm{cm}^{3}\right)\end{array}$ & PAR \\
\hline 0.00 & 0 & 265 & 265 & $4.54 \mathrm{E}+05$ & $3.27 \mathrm{E}+10$ & $9.49 \mathrm{E}+11$ & $\mathrm{n} / \mathrm{a}$ \\
\hline 0.07 & 20 & 54 & 31 & $5.59 \mathrm{E}+06$ & $5.33 \mathrm{E}+10$ & $1.06 \mathrm{E}+12$ & 0.06 \\
\hline 0.10 & 20 & 63 & 41 & $9.69 \mathrm{E}+06$ & $1.02 \mathrm{E}+11$ & $1.71 \mathrm{E}+12$ & 0.10 \\
\hline 0.14 & 20 & 64 & 48 & $1.12 \mathrm{E}+07$ & $1.16 \mathrm{E}+11$ & $1.67 \mathrm{E}+12$ & 0.13 \\
\hline 0.18 & 20 & 59 & 48 & $1.08 \mathrm{E}+07$ & $9.77 E+10$ & $1.25 \mathrm{E}+12$ & 0.17 \\
\hline 0.22 & 20 & 54 & 45 & $1.09 \mathrm{E}+07$ & $8.41 \mathrm{E}+10$ & $9.79 \mathrm{E}+11$ & 0.21 \\
\hline 0.26 & 20 & 49 & 41 & $1.20 \mathrm{E}+07$ & $7.96 \mathrm{E}+10$ & $8.81 \mathrm{E}+11$ & 0.25 \\
\hline 0.07 & 100 & 66 & 51 & $8.57 \mathrm{E}+06$ & $9.15 E+10$ & $1.27 \mathrm{E}+12$ & 0.32 \\
\hline 0.10 & 100 & 49 & 43 & $7.72 E+06$ & $5.03 \mathrm{E}+10$ & $5.32 \mathrm{E}+11$ & 0.48 \\
\hline 0.14 & 100 & 34 & 31 & $4.79 \mathrm{E}+06$ & $1.80 \mathrm{E}+10$ & $1.77 \mathrm{E}+11$ & 0.67 \\
\hline 0.18 & 100 & 28 & 21 & $4.18 \mathrm{E}+06$ & $1.13 \mathrm{E}+10$ & $9.43 E+10$ & 0.85 \\
\hline 0.22 & 100 & 25 & 17 & $1.59 \mathrm{E}+06$ & $4.33 \mathrm{E}+09$ & $6.37 \mathrm{E}+10$ & 1.04 \\
\hline 0.26 & 100 & $\mathrm{n} / \mathrm{a}$ & $\mathrm{n} / \mathrm{a}$ & $4.75 \mathrm{E}+04$ & $1.58 \mathrm{E}+09$ & $3.81 \mathrm{E}+10$ & 1.23 \\
\hline
\end{tabular}

Table 1: Data for single shot and multiple shot photofragmentation of soot particles. The total diameter is the mean diameter of the entire distribution of particles, while the small diameter is the mean for only the smallest mode of particles. 


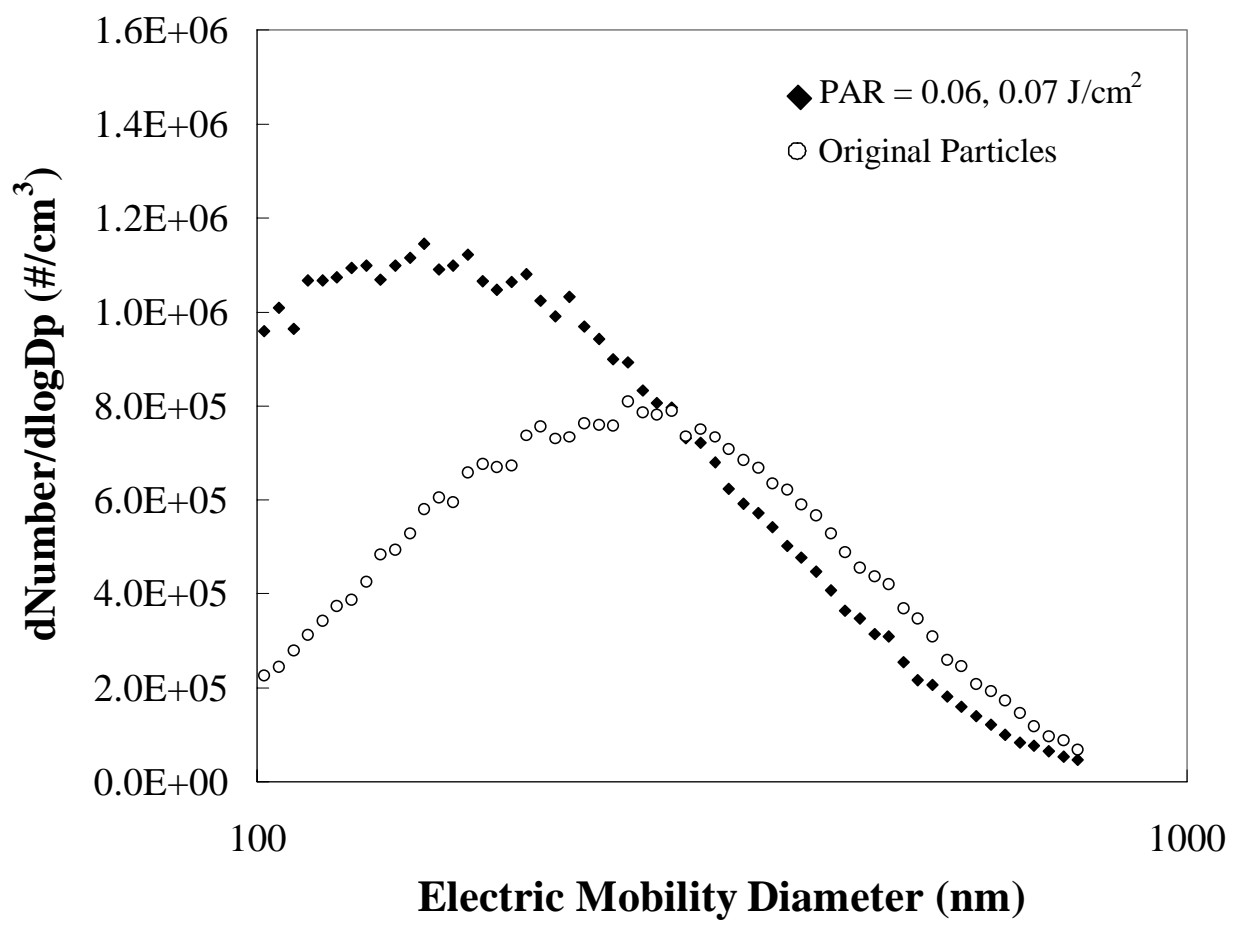

Figure 3: Comparison of non-irradiated particles and particles irradiated at a PAR of $0.06\left(0.07 \mathrm{~J} / \mathrm{cm}^{2}, 20 \mathrm{~Hz}\right)$. 


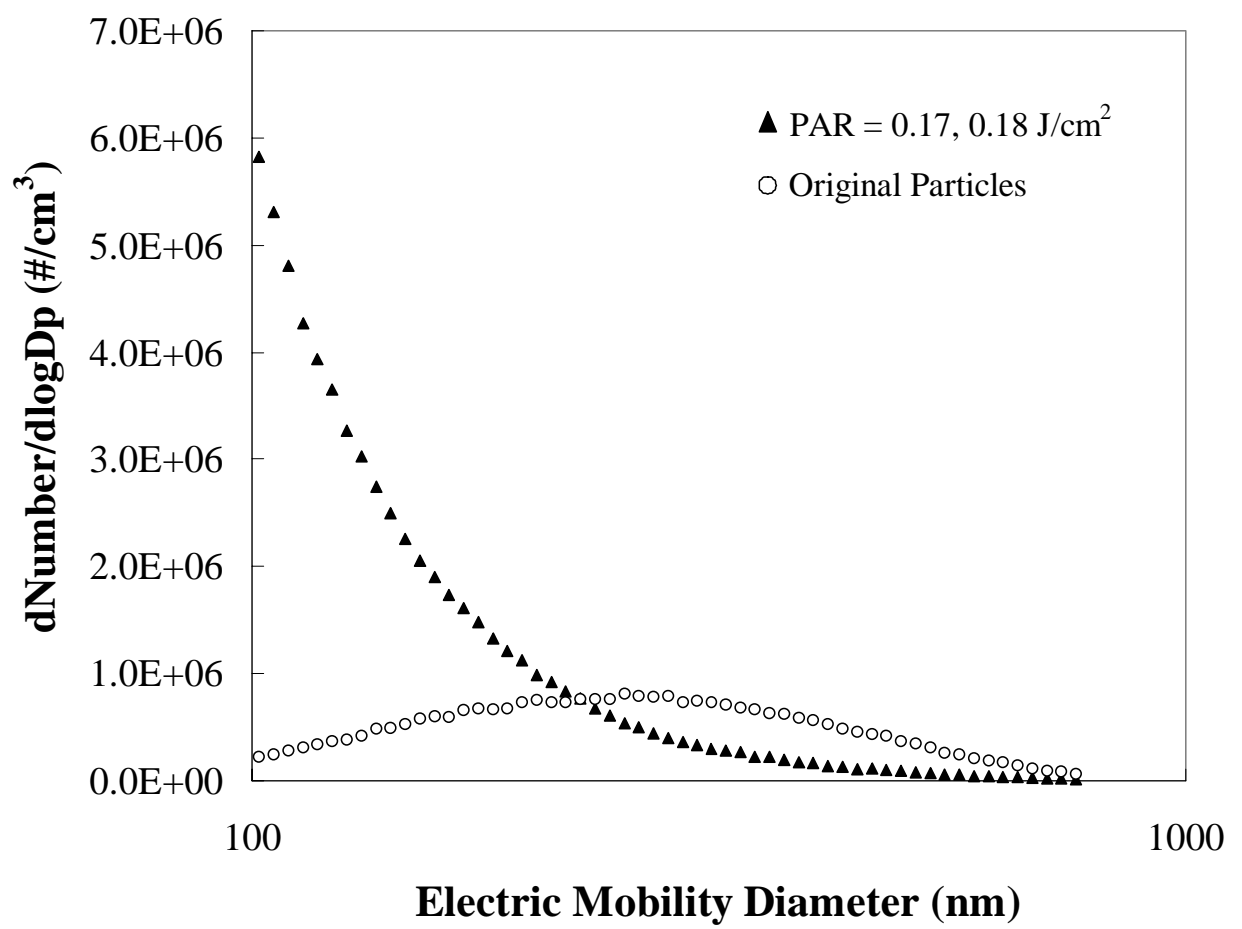

Figure 4: Comparison of non-irradiated particles and irradiated particles at a PAR of $0.17\left(0.18 \mathrm{~J} / \mathrm{cm}^{2}, 20 \mathrm{~Hz}\right)$. 


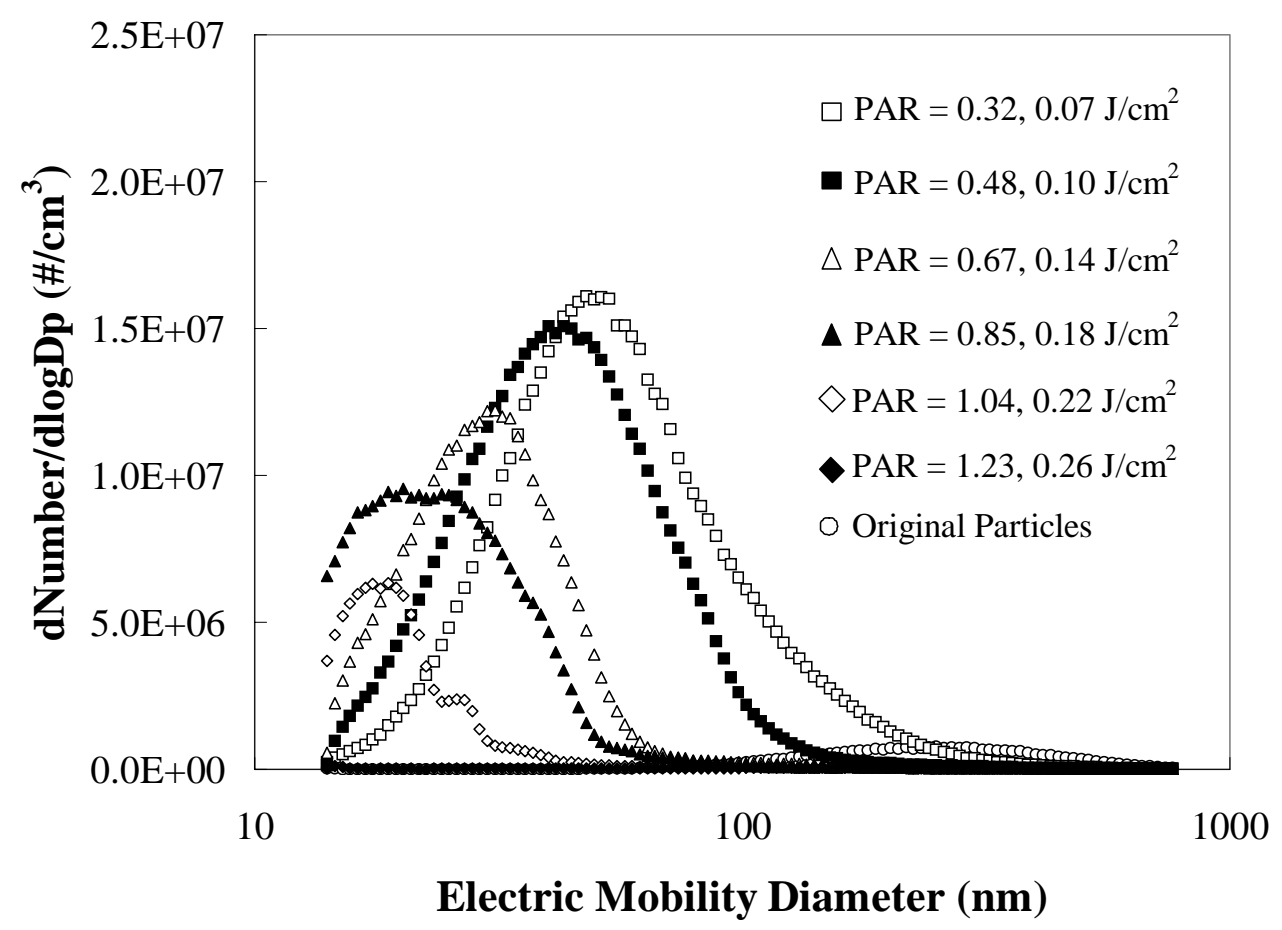

Figure 5: Size distributions of soot particles fragmented for PAR values from 0.32 to 1.23 (fluences from 0.07 to $0.26 \mathrm{~J} / \mathrm{cm}^{2}$ ) by $193 \mathrm{~nm}$ photons at $100 \mathrm{~Hz}$ (8 shots/particle). The original particle size distribution is shown in open circles. 


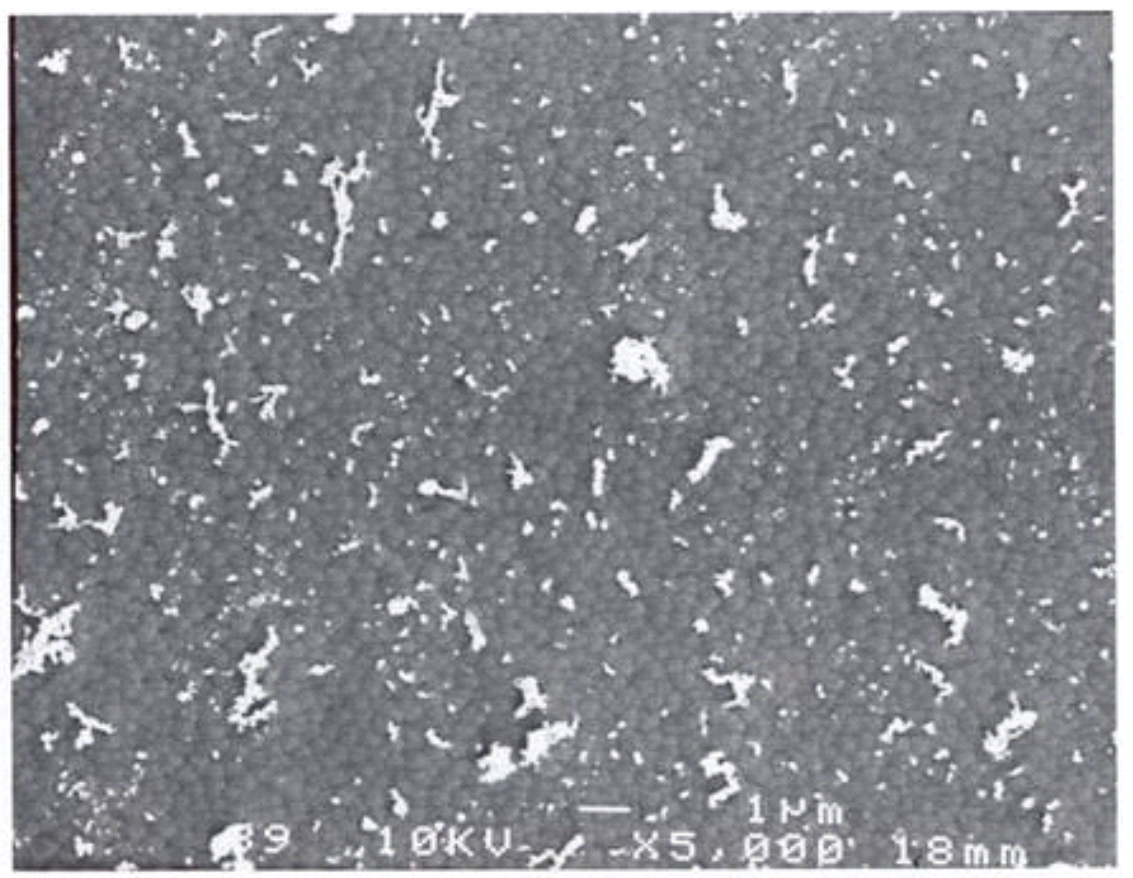

(a) Non-irradiated soot particles on a filter with a $20 \mathrm{~nm}$ filter pore size.

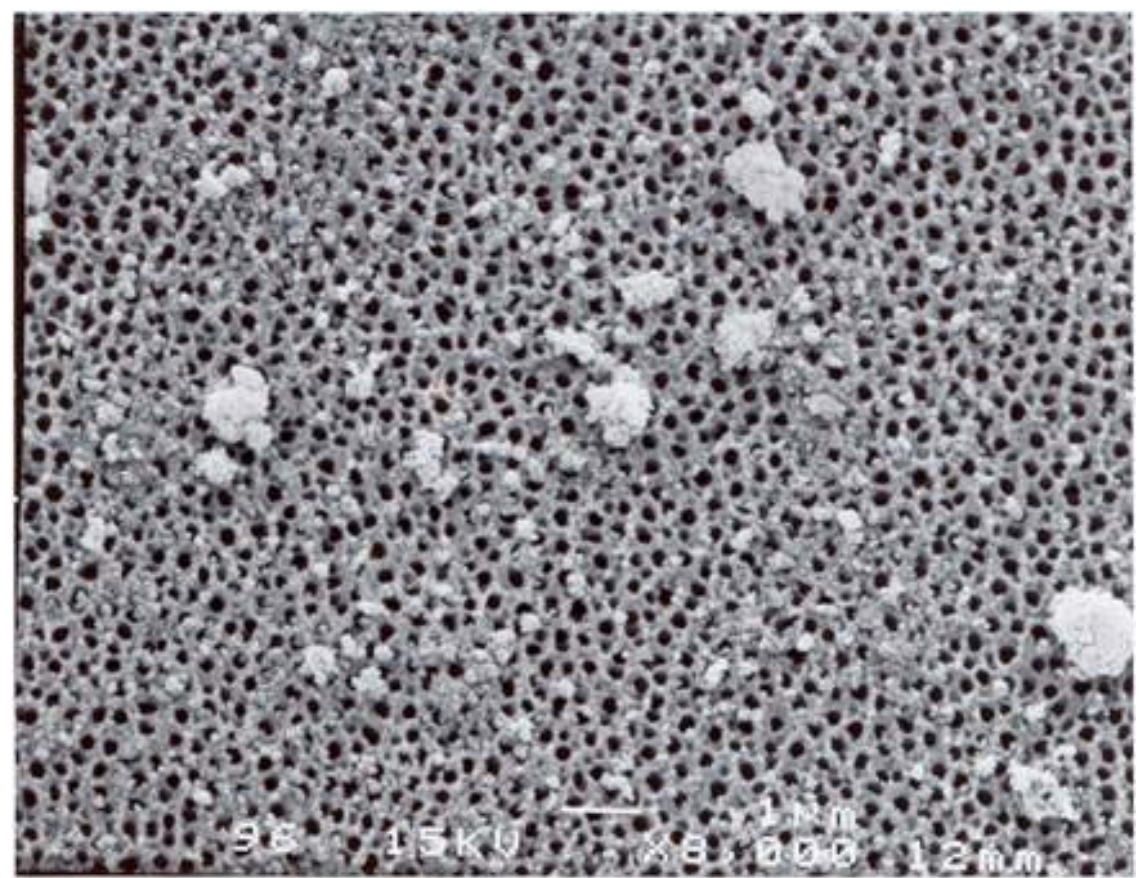

(b) Soot particles irradiated at a PAR of $0.85\left(0.18 \mathrm{~J} / \mathrm{cm}^{2}, 100 \mathrm{~Hz}\right)$. The filter pore size is $200 \mathrm{~nm}$.

Figure 6: SEM images of non-irradiated and irradiated soot particles. The non-irradiated particles are fractal agglomerates, while the irradiated particles are bi-modal. Many small primary particles are observed along with a few spherical agglomerates approximately $1 \mu \mathrm{m}$ in diameter. 


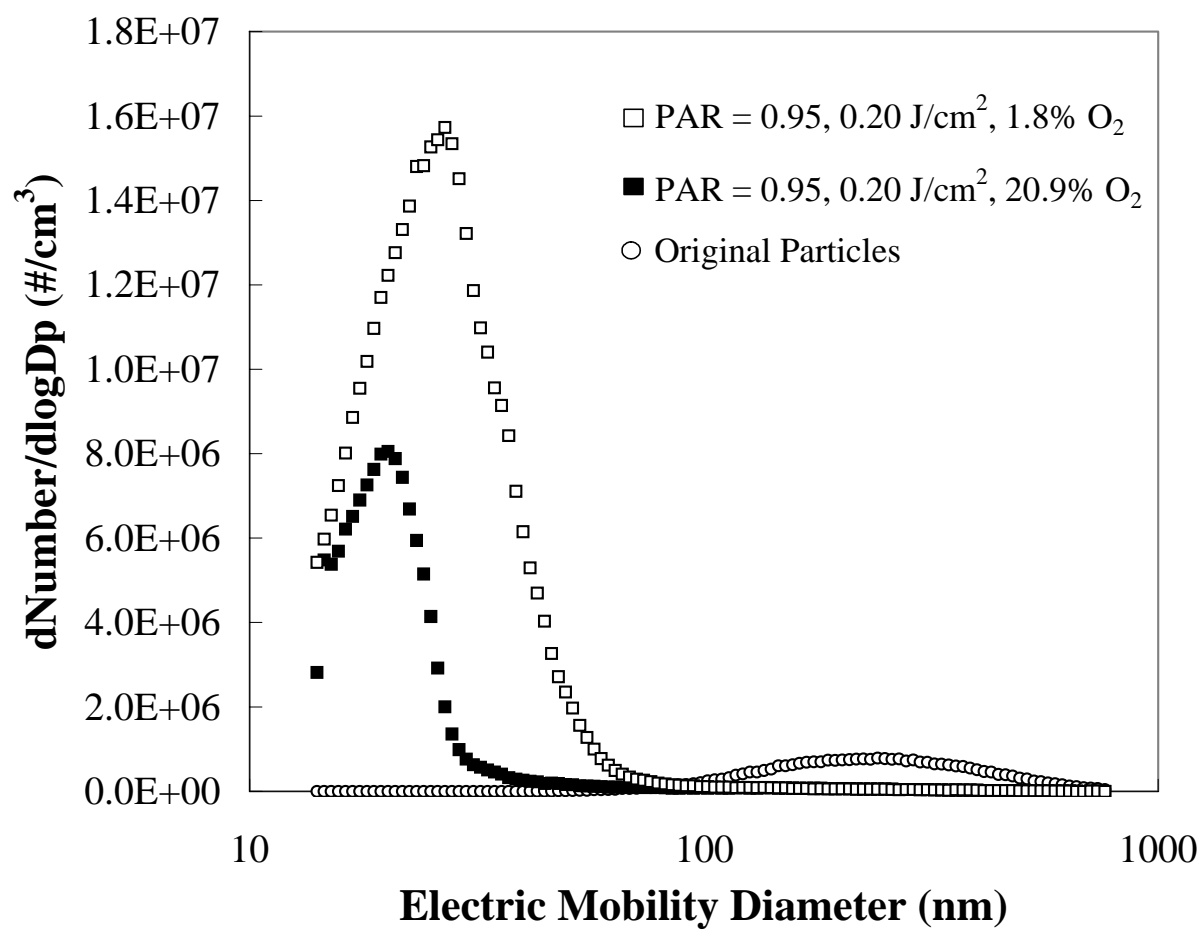

Figure 7: Size distributions of soot fragmented in exhaust gases diluted by $\mathrm{N}_{2}$ (open squares, $1.8 \% \mathrm{O}_{2}$ ) and air (filled squares, $20.9 \% \mathrm{O}_{2}$ ) at a PAR value of 0.95 (fluence of $0.20 \mathrm{~J} / \mathrm{cm}^{2}$ ) and $100 \mathrm{~Hz}$. The original particle size distribution is shown in open circles. Filter mass measurements of particles irradiated in air and $\mathrm{N}_{2}$ confirm that although the number concentrations vary by a factor of 3 , the volume concentrations are similar. 